\title{
Balkanologie
}

Balkanologie Revue d'études pluridisciplinaires

Vol. I, $n^{\circ} 1$ | 1997

Volume I Numéro 1

\section{La société, cette grande absente}

Essai critique sur les analyses occidentales du nationalisme postcommuniste

\section{Oliver Freeman}

Traducteur : Patrick Michels

\section{OpenEdition \\ Journals}

Édition électronique

URL : http://journals.openedition.org/balkanologie/192

DOI : 10.4000/balkanologie.192

ISSN : 1965-0582

Éditeur

Association française d'études sur les Balkans (Afebalk)

Édition imprimée

Date de publication : 1 juillet 1997

ISSN : $1279-7952$

Référence électronique

Oliver Freeman, «La société, cette grande absente », Balkanologie [En ligne], Vol. I, nº 1 | 1997, mis en ligne le 02 juin 2008, consulté le 17 décembre 2020. URL : http://journals.openedition.org/ balkanologie/192 ; DOI : https://doi.org/10.4000/balkanologie.192

Ce document a été généré automatiquement le 17 décembre 2020.

(c) Tous droits réservés 


\section{La société, cette grande absente}

Essai critique sur les analyses occidentales du nationalisme postcommuniste

\section{Oliver Freeman}

Traduction : Patrick Michels

\section{NOTE DE L'ÉDITEUR}

Ce texte a été traduit de l'anglais par Patrick Michels.

1 De nombreux articles de journaux et de rapports gouvernementaux consacrés aux difficultés de la transition post-communiste auxquelles sont confrontés les pays de l'Europe centrale et orientale développent des conclusions sinon contradictoires, du moins paradoxales dans leurs estimations de l'impact de l'expérience communiste sur les valeurs et les attitudes sociales. Cette influence est fréquemment reconnue lorsqu'on s'intéresse à des comportements tels que la discipline au travail ou le respect pour l'ordre légal personnifié par l'État. Par contre, les nombreux conflits qui perturbent cette région sont étiquetés "ethniques" et sont perçus comme une expression naturelle d'identités ancestrales "gelées" par quarante années de communisme. Adam Przeworski a justement suggéré que "toute explication rétrospective de la chute du communisme doit non seulement tenir compte du développement historique mais aussi identifier les hypothèses théoriques qui nous ont empêché d'anticiper ces développements ${ }^{1}$. Le but de cet article est de démontrer la valeur heuristique de cette remarque. D'abord, nous verrons que le paradoxe mentionné ci-dessus découle des tensions entre les deux principaux cadres intellectuels qui ont dominé dans les analyses occidentales des régimes communistes jusqu'en 1989 : le paradigme totalitaire et l'interprétation libérale du politique. Ensuite, nous soutiendrons que le totalitarisme et le libéralisme ont contribué au succès des deux "explications" dominantes $\mathrm{du}$ nationalisme post-communiste: la théorie $\mathrm{du}$ "gel communiste" et celle du "vide idéologique". Enfin, nous suggérerons que la recherche occidentale sur l'Europe centre-orientale a échoué à produire des analyses 
convaincantes sur les conflits d'identité qui ont émergé dans la région à cause de sa propension à recourir à ce qu'on pourrait appeler le "nationalisme méthodologique".

\section{L'obsolescence du paradigme totalitaire}

2 À la suite de la publication du travail séminal d'Hannah Arendt, Les origines $d u$ totalitarisme $e^{2}$, le paradigme totalitaire a été systématiquement appliqué à tous les États communistes. Ainsi que Daniel Nelson l'a souligné, «le regroupement des systèmes communistes soviétique, est-européen et asiatique a été initié par la politique étrangère des États-Unis et de l'Occident qui désignait ces États comme des adversaires, méritant un même traitement» ${ }^{3}$. Le "rideau de fer" fournissait une ligne de séparation commode entre une Europe occidentale pluraliste et démocratique et un "bloc soviétique", formé d'un centre maléfique, l'Union Soviétique, entouré d'un groupe d'États fantoches, les "démocraties populaires". Une nouvelle discipline - les études communistes, une nouvelle science- la soviétologie, une nouvelle théorie- le totalitarisme et le décor était planté pour que les étudiants du communisme forment une communauté isolée des tendances principales des sciences sociales ${ }^{4}$.

3 En synthétisant, le paradigme totalitaire soutient que le système communiste était essentiellement une structure du pouvoir du "haut vers le bas" dans laquelle une élite politique restreinte exerçait son pouvoir sur une masse passive d'individus isolés en imposant une conformité idéologique par la contrainte physique et la persuasion idéologique. Cette hypothèse implique trois corollaires. D'abord, que le changement ne peut être initié que du sommet de la hiérarchie politique. Ainsi les chercheurs se sentaient disposés à se concentrer presqu'exclusivement sur les élites. Les soviétologues sont progressivement devenus kremlinologues. Ensuite, que les différents régimes communistes présentaient plus de traits communs que de différences. Ils formaient un groupe spécial, appelé "bloc soviétique", "monde communiste", ou simplement "Europe de l'est". L'URSS, le "centre" du système international communiste, était le point de mire des recherches, tandis que d'autres pays sous direction communiste étaient négligés, comme si le changement dans la "périphérie" dépendait entièrement des décisions prises à Moscou. Enfin, que le communisme était irréformable. "Puisque les fonctions basiques de ce système social sont dirigées contre la société, qui, en retour, est privée de tout moyen constitutionnel d'auto-défense, le seul changement concevable doit donc prendre la forme d'une révolution violente. $»^{5} \mathrm{En}$ conséquence, le paradigme totalitaire était une théorie fermée, autant irréformable que l'objet qu'il avait construit. Sa logique interne déniait l'existence du pluralisme au sein des sociétés communistes, excluait que le changement puisse être induit en dehors de l'élite dirigeante et impliquait donc que les systèmes communistes ne pouvaient être renversés que par la force. Cette prophétie a été invalidée par la forme pacifique avec laquelle la plupart des élites communistes ont abandonné le pouvoir depuis 1989. Le succès de l'oxymoron "révolutions de velours" ou du néologisme "réfolution" traduit la déroute de nombreux observateurs occidentaux ${ }^{6}$. À la notable exception de la Roumanie, les événements de 1989 ont mis en lumière l'obsolescence du paradigme totalitaire' ${ }^{7}$. 


\section{L'échec de l'a-priori libéral}

4 À un niveau plus philosophique, le communisme, en tant qu'idéologie et système politique, fut projeté comme l'antithèse de ce qui constitue la principale prémisse de la pensée libérale, à savoir l'individualité et l'unicité de l'homme. Il était supposé que la conformité dans les sociétés communistes était maintenue par le seul recours à la force brute et que derrière la façade il demeurait un incompressible désir de liberté. Comme l'écrit Andrzej Korbonski, "l'explication générale du succès communiste était, à quelques exceptions près, dépeinte en noir et blanc, avec la maléfique Union Soviétique impérialiste utilisant simplement son impressionnante puissance militaire pour détruire l'amour de la liberté des Européens de l'est $»^{8}$. Tout signe de dissidence, par des individus isolés, des groupes sociaux, ou des mouvements de masse était immédiatement salué sous le drapeau des idées libérales et des droits de l'Homme. Le problème était que l'école occidentale, avec son engouement pour le modèle totalitaire, était incapable d'apprécier la pleine signification de telles manifestations de pluralisme. Intégrer le pluralisme dans le cadre du paradigme totalitaire signifiait, sinon son auto-négation, du moins une extension conceptuelle telle que le pouvoir discriminant du terme disparaissait ${ }^{9}$. Les libéraux, quant à eux, induisaient des croyances sociales des idées professées par quelques individus isolés. Les Soviétiques devenaient des Sakharov terrifiés, les Tchécoslovaques des Havel effrayés et les Polonais des Michnik épouvantés. Lorsque, finalement, les régimes communistes se désintégrèrent en 1989, la réaction initiale fut de supposer une sorte de tabula rasa, dans l'espoir que l'esprit de liberté et de démocratie serait débridé et jouerait comme une main invisible guidant les populations dans leur transition du monde du "mal" vers celui du "bien". En Occident, beaucoup crurent que les populations à l'est du "rideau de fer" de victimes passives de l'ordre totalitaire deviendraient automatiquement des partisans actifs de la démocratie, de l'économie de marché et des droits de l'homme. Ainsi que le montrèrent d'une manière flagrante les événements qui suivirent, ces voeux pieux cédèrent rapidement la place au désenchantement.

5 La combinaison du paradigme totalitaire et de la pensée libérale conduisit à une situation dans laquelle la capacité structurale de l'État était perçue comme la négation des aspirations culturelles des individus et vice-versa. Tout comme le paradigme totalitaire fut renversé par l'écroulement pacifique des États communistes, le biais libéral était incapable d'expliquer pourquoi les sociétés s'identifiaient aux notions particularistes d'ethnicité et de nationalité plutôt qu'aux idéaux universalistes de la démocratie libérale et des droits de l'Homme. Ces deux échecs tenaient essentiellement au fait que ni le paradigme totalitaire, avec son insistance sur l'État tout-puissant, ni le libéralisme, avec son principal intérêt pour les individus, n'ont suffisamment porté leur attention sur les transformations qui ont eu lieu durant la période communiste au niveau intermédiaire, celui de la société. Cette omission trouva son corollaire dans les deux thèses dominantes qui ont été avancées pour expliquer l'essor des conflits ethniques après la chute du communisme : la théorie du "gel communiste" et celle du "vide idéologique".

\section{La théorie du « gel communiste »}

6 En novembre 1989, Vaclav Havel affirma que «l'histoire, qui avait été artificiellement arrêtée, s'est remise en route $»^{10}$. Les titres de nombreux articles ou de monographies 
publiés par la suite véhiculèrent cette image du communisme comme une simple parenthèse de l'histoire ${ }^{11}$. La théorie du "gel communiste" suit la même logique dans ce sens qu'elle postule que le communisme a "gelé" toutes les haines ethniques de l'Europe et de l'empire russe pré-communistes. L'éruption des conflits ethniques dans l'Europe post-communiste est assimilée à un génie relâché d'une bouteille hermétiquement close après quarante années de confinement dans le carcan totalitaire $^{12}$. Des journalistes, comme Neal Ascherson affirmaient que «les litiges nationaux et ceux avec les minorités n'ont pas été résolus par le communisme mais simplement enfermé dans le congélateur de l'État policier $»^{13}$. Des universitaires comme André Liebich écrivaient que «le communisme n'a pas été la couveuse du malaise minoritaire mais, tout au plus, le congélateur dans lequel ce malaise est resté conservé $»^{14}$. Tout comme dans l'exposé de Vaclav Havel, l'hypothèse sous-jacente de ce vocabulaire métaphorique est que l'histoire a été immobile durant la période communiste. À y regarder de plus près, la comparaison du communisme à un congélateur des haines ethniques pose en fait plus de problèmes qu'elle n'en résout.

7 Tout d'abord, elle implique que quatre décennies d'administration communiste n'ont eu aucun effet sur les identités subjectives des populations concernées. Sous l'influence du paradigme totalitaire, de nombreux universitaires ont essentiellement centré leur attention sur les modifications institutionnelles décidées au sommet et négligé l'étude des valeurs dominantes au sein de la société. Les informations sur les dirigeants politiques étaient relativement faciles à obtenir, tandis que les données empiriques, notamment les sondages, à propos des attitudes et croyances sociales étaient soit indisponibles, soit écartées comme étant idéologiquement biaisées. Plutôt que de se préoccuper de l'histoire politique et sociale de la période communiste, de nombreux soit-disants "spécialistes" de l'Europe de l'est ont utilisé la métaphore du "gel communiste" comme un raccourci commode, un écran de fumée servant à masquer, d'une part, leur manque d'intérêt dans le passé de cette région, et, d'autre part, leur ignorance des sociétés actuelles. Cela leur a permis de s'intégrer rapidement aux programmes de recherches établis par des gouvernements occidentaux dans leur quête pour influencer le cours des événements dans l'Europe orientale contemporaine. Au lieu de renoncer à leur passé et de réviser leurs manuels, de nombreux anciens soviétologues se sont tournés vers l'industrie plus lucrative de la "transitologie" et ont abandonné la nécessaire ré-interprétation du passé communiste aux historiens ${ }^{15}$.

La seconde principale critique que l'on peut formuler à l'adresse de la théorie du "gel communiste" est qu'elle réinstitue la division artificielle entre l'est et l'ouest. La distinction établie durant la guerre froide entre les régimes "totalitaire" et "démocratique" est remplacée par la division entre le "retard" et la "modernité" ${ }^{16}$. À cet égard, il est remarquable que l'Occident ait été aussi unifié pour condamner les conflits ethniques comme un retour à la barbarie. Ainsi que l'écrit Katherine Verdery, la théorie du "gel communiste" "combine avec une vision apparente de la période socialiste comme, sous tous rapports, une aberration dont la fin restaure le commerce comme une norme, un ordre plus normal des passions "tribales irrationnelles" dans une partie du monde qui a longtemps été perçu comme attardé ${ }^{17}$. Ivan Volgyes, par exemple, se réfère à un "tribalisme régénéré", à des "atavismes d'un âge révolu", ou à un "retour aux modèles primitifs de comportement national"18. L'affirmation implicite qui se profile derrière une telle terminologie est que l'Europe orientale a quarante ans de retard par rapport à l'Occident dans la marche inexorable de l'humanité vers le 
progrès. Cette vision du retard peut être vue dans l'attitude paternaliste de l'Occident envers cette région. En même temps, ainsi que Katherine Verdery le souligne plutôt cyniquement, elle "peut servir aux politiciens nationalistes de l'Europe orientale de moyen de justifier leurs actions et aux décideurs occidentaux de justification de leur inaction $»^{19}$. En maintenant la dichotomie est-ouest, la théorie du "gel communiste" ne fournit aucune explication du gain de popularité simultané des idéologies fascistes et racistes en Europe occidentale.

9 La troisième insuffisance de cette approche est qu'elle tend à expliquer tout en terme d'ethnicité ou de nationalisme. Ainsi que Lynne Jones le note, «emballer tous les développements négatifs qui ont eu lieu dans le monde post-communiste depuis 1989 de cette façon semble, d'une certaine manière, réduire la frustration et la déception que 1989 n'ait pas rapidement pris le chemin que nous souhaitions $»^{20}$. La fixation occidentale sur l'ethnicité en tant que racine du problème a eu comme résultat désastreux la tentative d'imposer la "cantonisation" de la Bosnie-Herzégovine sous la forme du plan Vance-Owen. Une autre conséquence est d'attribuer systématiquement un sens négatif aux termes d'ethnicité et de nationalisme, lorsqu'ils s'appliquent à l'Europe orientale. Ce qui revient à ignorer que le nationalisme fut aussi une des grandes sources d'inspiration de l'opposition démocratique dans ces pays, et qu'il contribua à nourrir un sentiment de communauté qui encourageait la justice sociale et l'aide mutuelle. Finalement, en réduisant les formes contemporaines de nationalisme intégral en Europe centre-orientale à l'expression d'un nationalisme "latent" qui a traversé intact la période communiste, l'approche du "gel communiste" réduit le rôle que d'autres facteurs objectifs et subjectifs ont joué durant la période communiste en précipitant son aboutissement ${ }^{21}$.

10 La théorie du "gel communiste" a rencontré extrêmement de succès et demeure très à la mode aujourd'hui. Elle fournit une explication apaisante à une apparente évolution irrationnelle des sociétés post-communistes parce qu'elle rejette la faute sur leur propre "arriération" plutôt que sur l'incapacité de l'Occident à prévoir, et par conséquent neutraliser, les forces libérées par le nationalisme intégral. Imaginer que les identités nationales d'avant-guerre soient suffisamment anciennes et établies pour survivre intactes à l'interlude communiste et hiberner en attendant le "printemps des nations" est une interprétation ethnocentrique qui ne résiste pas à l'inspection historique $^{22}$. Non seulement le processus d'édification nationale et étatique était différent dans l'Europe occidentale et centre-orientale avant la prise du pouvoir communiste, mais aussi la souveraineté limitée imposée par l'URSS alimenta les sociétés de l'Europe centre-orientale de sentiments anti-impérialistes qui s'adressaient aussi bien à la domination russe qu'à la distribution formelle du pouvoir entre les groupes ethniques qu'elle avait entraînées dans chaque pays.

\section{La théorie du « vide idéologique »}

11 Selon la théorie du "vide idéologique", «la faiblesse de la société civile, la longue absence de débat politique (...) ont laissé un vide idéologique et éthique - aisé à combler avec des préjugés nationalistes et racistes ${ }^{23}$. Tandis que sous le pouvoir des communistes les populations étaient habituées à un sentiment d'inertie politique et de fatalité historique, elles furent soudainement mises en face d'une situation d'agitation politique, sociale et économique avec de nouvelles directions qui n'étaient plus proposées par une autorité clairement définie, le Parti communiste, mais par 
différentes élites, héritères ou opposées à l'ancien système. La brève euphorie qui suivit immédiatement la désintégration des régimes communistes céda rapidement la place à un sentiment d'insécurité et de désorientation. Les gens étaient appelés à re-construire une identité commune pour affronter les incertitudes du futur. La "nation" fournit l'identité et le nationalisme la direction. En conséquence, le nationalisme combla le fossé idéologique entraîné par la disparition du régime communiste. Comme l'approche $\mathrm{du}$ "gel communiste", le succès de la théorie du "vide idéologique" peut être expliqué par sa simplicité.

Premièrement, elle soutient que le nationalisme a succédé au communisme, comme s'il y avait une nette séparation entre les deux. C'est négliger le fait que le nationalisme a joué un grand rôle non seulement dans l'imposition mais aussi dans l'effondrement du communisme. Elle exclut la possibilité qu'il y ait eu d'autres alternatives à la désintégration des systèmes communistes le long des lignes ethniques. En réalité, c'était plutôt lié au degré d'homogénéité ethnique qu'à l'expérience communiste en elle-même. Des États multi-ethniques tels que la Yougoslavie, la Tchécoslovaquie ou l'Union Soviétique, se sont déchirés et ont éclaté en plus petits États prétendant à l'unité ethnique. Des pays avec d'importantes minorités, tels que la Roumanie ou la Slovaquie, paraissent être sous une constante menace de dissension interne. Enfin, dans des États ethniquement homogènes, tels que l'Albanie ou la Pologne, le nationalisme est resté remarquablement absent du débat politique interne. La théorie du "vide idéologique" ne peut donc être appliquée sans discrimination à tous les pays qui ont vécu l'expérience du régime communiste.

Deuxièmement, la théorie du "vide idéologique" suggère que les nationalismes communiste et post-communiste sont deux phénomènes comparables qui remplissent la même fonction idéologique et éthique dans la mesure où les deux fournissent à la population des cadres de référence qui lui permettent de définir ses relations avec le monde "du dedans" et celui "du dehors". Cependant, tandis que le nationalisme est une forme largement spontanée d'intégration sociale culturellement déterminée qui existe simplement du fait que le peuple y croit, le communisme, en tant que théorie d'intégration sociale de classe, était essentiellement la doctrine d'un système politique qui ne rendait qu'un hommage peu sincère à la philosophie originelle de Marx. Pour cette raison, c'était plus l'attitude du peuple envers le système politique tel qu'il fonctionnait en pratique qui fut la source d'inspiration des attitudes de soutien, soumission, indifférence, compromis ou rejet, l'élément de foi consciente ou de croyance dans l'idéal communiste n'étant en fait que secondaire. En conséquence, la théorie du "vide idéologique" ne parvient pas à distinguer les attitudes des croyances d'une part, et l'État communiste de la nation comme source ou récipient de telles attitudes ou croyances d'autre part.

Les deux théories, celle du "gel communiste" et celle du "vide idéologique", ignorent la possibilité que quelques stimuli ayant précipité l'émergence du nationalisme après 1989 aient été amplifiés, sinon créés, par l'expérience communiste elle-même. La primauté accordée aux facteurs externes est aussi affirmée par des universitaires qui soutiennent que la cause réside essentiellement dans l'esprit de revanche antiimpérialiste qui a succédé à l'écroulement de l'empire soviétique ${ }^{24}$ et qui fut alimenté par les récents événements tels que l'état d'alerte écologique consécutive au désastre de Tchernobyl, la peur engendrée par l'intensification du flux démographique dans les années 1980 ou l'onde de choc produite par la révolution médiatique lancée par la 
glasnost de Gorbatchev ${ }^{25}$. De telles explications ne fournissent que peu d'éclairage sur la raison pour laquelle l'écho populaire qu'ont connu ces développements dans les sociétés de l'Europe centre-orientale a pris la forme du nationalisme.

\section{Les dangers du nationalisme méthodologique}

15 Aussi bien l'approche du "gel communiste" que la théorie du "vide idéologique" sont influencées par ce que l'on peut appeler le "nationalisme méthodologique". En fait, les deux utilisent le concept "nation" aussi bien en tant qu'objet d'analyse que comme principal facteur explicatif. Du point de vue méthodologique, les sciences sociales impliquent l'existence d'une unité d'analyse, le groupe social. Les membres d'un même groupe doivent partager certaines caractéristiques communes qui ne peuvent être réduites à la somme des caractéristiques de chaque membre pris isolément ${ }^{26}$. Le principal problème est de savoir sous quelles conditions un individu peut être classé comme appartenant à un groupe plutôt qu'à un autre. La question se pose, verticalement, lorsqu'on tente de déterminer si un individu est représentatif d'une culture, d'une nation, d'une région ou d'un clan ${ }^{27}$. Horizontalement le problème se pose lorsqu'on cherche à classer un individu dans une classe sociale, un groupe ethnique ou une tribu. Dans leur réponses à de telles questions, la principale ligne de démarcation se situe entre les scientifiques qui recourent aux critères objectifs ou externes de différenciation et ceux qui attachent une importance primordiale à l'identification subjective. Dans la plupart des cas, chaque individu appartient à un, et un seul seulement, de ces groupes. À cet égard, la majeure partie de la littérature sur le nationalisme tombe en proie aux muses du nationalisme. La nation est acceptée comme une réalité donnée d'où sont déduits les traits communs à tous ses membres. Cette inversion de logique aboutit à une vision conservatrice des nationalités existantes encline à maintenir le statu quo d'une part (l'approche du "gel communiste"), ou à oublier les changements survenant au sein et entre chacune de ces communautés d'autre part (la théorie du "vide idéologique"). Ce nationalisme méthodologique soustend la plupart de la littérature sur le nationalisme contemporain en Europe centreorientale.

Il faut souligner que la conscience nationale est un phénomène de masse et non pas d'élite. Le problème est que ce sont les élites qui ont enregistré l'histoire et produit les preuves sur lesquelles de nombreux universitaires s'appuient dans leurs analyses du nationalisme. La supposition que les élites rendent comptent nécessairement des sentiments exprimés au niveau de la population est le reflet plus d'une prédilection que de la réalité. Ainsi qu'Eugen Weber l'a montré, il a fallu plus d'un siècle pour que la masse des paysans français se perçoivent en tant que membres de la nation française ${ }^{28}$. Les sondages réalisés auprès des émigrés polonais aux États-Unis entre 1840 et 1915 montrent que la majorité d'entre eux ne se considéraient pas en tant que Polonais mais en tant que Silésiens, Gorals ou Kachubiens ${ }^{29}$. Parler de Français avant 1870 ou de Polonais avant 1915 revient à induire la nationalité de la citoyenneté dans le premier cas et de la langue ou de la culture dans le second. Le nationalisme méthodologique, en accordant une importance exagérée à la pertinence historique des notions artificielles de "francité" et de "polonité", contourne la difficulté de surmonter le décalage entre la différentiation objective et l'identification subjective d'une part et l'inadaptation entre les revendications des élites et les croyances populaires d'autre part. 
17 Une autre conséquence du nationalisme méthodologique est que les études sur l'évolution du nationalisme dans les pays communistes se sont presqu'exclusivement concentrées sur des cas où les groupes ethniques semblaient faciles à identifier. Il y a une abondance de travaux universitaires sur les États multinationaux tels que l'URSS, la Tchécoslovaquie ou la Yougoslavie, ou sur les politiques officielles envers les minorités nationales comme les Hongrois en Roumanie ou les Turcs en Bulgarie. Par contre, la tension interne entre la conscience nationale et le socialisme d'État dans des États plus homogènes ethniquement, tels que la Pologne ou l'Albanie, n'a guère été analysée ${ }^{30}$. $C^{\prime}$ est comme si la pureté ethnique rendait l'étude du nationalisme non pertinente ${ }^{31}$. Le peu d'études qui ne souscrivent pas à cette vue se concentrent principalement sur l'impact des traditions "nationales" sur les régimes communistes, sans se préoccuper de l'influence des seconds sur les premières ${ }^{32}$. De plus, elles ignorent la possibilité que, dans des États communistes ethniquement homogènes, il puisse y avoir des définitions concurrentes de la "nation", qui, sans suivre nécessairement des lignes ethniques, exercent néanmoins une importante influence sur l'évolution de l'identification nationale dans ces pays.

\section{Conclusion}

18 L'échec de la socialisation politique communiste est généralement admis. En Occident, un tel verdict renforce la croyance dans la supériorité ultime du libéralisme sur des idéologies égalitaires. En Europe centrale, il sert à proclamer une pureté précommuniste ternie par plus de quarante ans de socialisme d'État. Malheureusement pour tous, les grands espoirs nés en 1989 avec ce que Zbigniew Brzezinski a baptisé "le Printemps des nations" se sont évaporés dans la chaleur d'une violence inconnue en Europe depuis la seconde guerre mondiale. Bien qu'il y ait plusieurs explications différentes du nationalisme post-communiste, il est hors de doute que les théories du "gel communiste" et du "vide idéologique" dominent le débat. Ceci peut être expliqué par leur apparente simplicité. En fait, les deux, bien que de manière différente, correspondent aux perceptions simplifiées de l'Europe centre-orientale qui ont prévalu en Occident avant l'écroulement du communisme. Ainsi que l'histoire l'a révélé depuis la chute des régimes communistes en Europe de l'est, il est devenu évident que les paradigmes dominants depuis la guerre froide ont un urgent besoin d'être révisés.

19 S'il est vrai que le passé explique les conditions actuelles, il est également vrai que le présent crée le passé. Les débats passés et présents à propos des relations entre le communisme et le nationalisme l'illustrent bien. Considérés comme des idéologies politiques isolées, le communisme et le nationalisme sont philosophiquement incompatibles. Tandis que le premier recommande la transformation sociale et la différentiation horizontale le long des lignes de classe, le second accentue la continuité historique et les principes, verticalement unifiants, d'une communauté immémoriale, atavique: la "nation". En pratique, cependant, un régime politique purement communiste, ou purement nationaliste, n'a jamais existé. Tous les partis communistes qui ont saisi le pouvoir en Europe centre-orientale après 1945 ont fait appel au nationalisme afin de tempérer l'opprobre populaire. Chaque parti promouvait son propre "communisme national" qui proposait une vision différente de la nature et des objectifs de la communauté nationale à celle qui avait existé avant la seconde guerre mondiale. Comme en URSS après 1917, le nationalisme était une partie intégrante du communisme appliqué en Europe de l'est après 1945. Á cet égard, 1989 ne constitua pas 
une tabula rasa mais plutôt un changement des formes dans lesquelles les aspirations nationales pouvaient s'exprimer. 1989, la soi-disante annus mirabilis, ne constitua pas non plus un retour au statu quo ante. En imposant une complète occultation de la phase communiste, ces deux formules évitent la principale question qui est de savoir à quel point les aspirations nationales ont été transformées par une expérience politique qui a embrassé deux générations.

En conclusion, cet article soutient que pour arriver à une meilleure compréhension de ce qui s'est déroulé et de ce qui se passe en Europe centre-orientale, il est nécessaire de remettre en question le biais nationaliste des travaux universitaires traditionnels et de s'interroger davantage sur l'influence que l'expérience communiste a exercé sur la conscience nationale. En effet, les différentes formes qu'a pris le nationalisme depuis la chute du communisme dérivent non seulement des "traditions nationales", mais aussi des facteurs "systémiques" qui se sont développés avec l'expérience communiste. Il faut donc considérer les effets du socialisme d'État sur le sentiment positif d'appartenance et le sentiment négatif d'exclusion, non seulement par rapport à la nation, mais aussi à l'État, au système communiste et aux différentes classes sociales. Maintenant que les archives sont ouvertes et que les gens sont libres de s'exprimer, il est grand temps d'écrire la vraie histoire de l'expérience communiste, en bref de réintégrer la dimension sociale dans un discours dominé jusqu'à présent par l'obsession du politique. Renoncer à cet effort ne fera que prolonger la détresse des universitaires occidentaux face au nationalisme post-communiste.

\section{NOTES}

1. Przeworski (Adam), "The "East" Becomes the "South" ? The "Autumn of the People" and the Future of Eastern Europe », Political Science and Politics, 24 (1), mars 1991, p. 20.

2. Arendt (Hannah), The Origins of Totalitarianism, New York: Harcourt and Co, 1951.

3. Nelson (Daniel L.), "Comparative Communism : A Postmortem », in Taras (Raymond C.), ed., Handbook of Political Science Research of the USSR and Eastern Europe. Trends from the 1950s to the 1990s, Westport / London : Greenwood Press, 1992, p. 308.

4. Sur ce point, voir Collignon (Jean-Guy), « De l'isolationnisme au comparatisme (méthodes et approches anglo-saxonnes pour l'analyse du système politique soviétique) », Revue Française de Science Politique, 26 (3), juin 1976.

5. Kolakowski (Leszek), « Hope and Hopelessness », Survey, 17 (3), 1971.

6. Voir les deux essais de Ash (Timothy Garton), « Reform or Revolution », « Refolution », dans The Uses of Adversity, London : Granta Books, 1989.

7. Il y a eu de nombreuses tentatives, surtout après 1956, d'introduire une vision plus comparative en utilisant les concepts ou les théories systémiques ou développementalistes tels que groupes d'intérêt, bureaucratie, corporatisme, société civile de culture politique. Néanmoins, les prémisses du paradigme totalitaire ont continué à servir de fondement à l'écrasante majorité des travaux sur les pays communistes. Cf. Tarschys (Daniel), «The Soviet Political System: Three Models », European Journal of Political Research, 5, 1977 ; Korbonski (Andrzej), « The Decline 
and Rise of Pluralism in East Central Europe, 1949-1989, or How Not to See the Forest for the Trees ", Communist and Post-Communist Studies, 26 (4), décembre 1993.

8. Korbonski (Andrzej), art.cit., p. 240.

9. Voir par exemple Kassof (Allen), «Totalitarianism without Terror », World Politics, 16, 1964 ; Ritvo (Herbert), "Totalitarianism without Coercicion?», in Brumberg (Abraham), ed., Russia under Krushchev. An Anthropology from Problems of Communism, New York : Praeger, 1962. Sur l'idée d'extension conceptuelle, voir Sartori (Giovanni), «Concept Misformation in Comparative Politics ", American Political Science Review, 64, 1970.

10. Cité par Rupnik (Jacques), «Eisschrank oder Fegefeuer. Das Ende des Kommunismus und das Wiedererwachen der Nationalismen in Osteuropa », Transit, 1, automne 1990, p. 132.

11. Glenny (Misha), The Rebirth of History, Harmondsworth : Penguin, 1990 ; Brossat (Alain), Combe (Sonia), Potel (Jean-Yves), Szurek (Jean-Charles), éds., A l'Est la mémoire retrouvée, Paris: La Découverte, 1990; Rothschild (Joseph), Return to Diversity, New York: Oxford University Press, 1989; Roskin (Michael), The Rebirth of Eastern Europe, Englewood Cliffs : Prentice Hall, 1991.

12. Sur ce point, voir Smith (Anthony D.), « Gastronomy or geology? The role of nationalism in the reconstruction of nations ", Nations and nationalism, 1 (1), mars 1995.

13. Ascheron (Neal), « Old wounds Exposed by the Post-Communism Thaw », The Independent on Sunday, 06/10/91.

14. Liebich (André), "À la recherche d'une solution introuvée », in Liebich (André), Reszler (André), éds., L'Europe centrale et ses minorités: vers une solution européenne ?, Paris: Presses Universitaires de France, 1993, p. 196. Dans une formulation plus ambiguë, Daniel-Louis Seiler soutient que «l'Union Soviétique a mis la question ethnico-nationale au frigidaire, en oubliant d'ajouter de la glace. La porte est maintenant grande ouverte », Seiler (Daniel-Louis), « InterEthnic Relations in East Central Europe : The Quest for a Pattern of Accomodation ", Communist and Post-Communist Societies, 26 (4), décembre 1993, p. 352.

15. Ainsi que l'écrit Miroslawa Marody, «le principal problème que les réformateurs doivent reconnaître est lié au fait que les actions quotidiennes des individus seront déterminées par les habitudes acquises au cours des expériences sociales radicalement différentes de celles qu'auraient rempli de nouvelles institutions». Marody (Miroslawa), «Dylematy postaw politycznyh i orientacji swiatopogladow », in Wiatr (Jerzy J.), éd., Polska 1980-1990, Warsaw : Warsaw University Press, 1990, p. 167, n. 5.

16. Dans l'Europe centre-orientale, la distinction s'opère entre "communisme" ou "socialisme" d'une part et "civilisation", "normalité" ou "européanité" de l'autre.

17. Verdery (Katherine), "Nationalism and nationalist sentiment in post-socialist Romania ", Slavic Review, 52 (2), 1993, p. 179. Dans son article, Verdery fait référence à la théorie des "haines ancestrales".

18. Volgyes (Ivan), "Military security in the post-communist age : reflections on myths and realities", Studies in Comparative Communism, 25 (1), 1992. Sur cette question, voir Ranger (Terence), « Tribalisation of Africa. Retribalisation of Europe », Woodstock Road Editorial. A Oxford Magazine of International Affairs, Oxford : St Anthony's College, (16), 1994.

19. Verdery (Katherine), art.cit., p. 180, n. 5.

20. Jones (Lynne), « Nationalism and the Self », Peace and Democracy News, hiver 1992-1993, p. 20.

21. Ce point est soulevé par Raymond Pearson bien qu'il ne tienne pas compte des facteurs subjectifs. Voir Pearson (Raymond), "The making of ' 89 : nationalism and the dissolution of communist Eastern Europe », Nations and Nationalism, 1 (1), 1995.

22. Ceci a donné lieu à de nombreuses controverses entre les spécialistes du nationalisme ouestet est-européens. Voir par exemple Gellner (Ernest), «Nationalism and Politics in Eastern Europe ", New Left Review, (189), 1991 ; Magaš (Branka), « Nationalism and Politics in Eastern Europe : A Response to Ernest Gellner », New Left Review, (190), 1991. 
23. Kaldor (Mary), « Citizens' Democracy Builds Peace », Yugofax, (8/9), december 1991, d'après Jones (Lynne), art.cit., p. 22.

24. Voir par exemple Schöpflin (George), Politics in Eastern Europe, 1945-1992, Oxford : Blackwell, 1993 ; Swain (Geoffrey), Swain (Nigel), Eastern Europe since 1945, London : Macmillan, 1993.

25. Sur ce point, voir Pearson (Raymond), op.cit., p. 69-80.

26. Cette loi fondamentale des sciences sociales a d'abord été formulée par Durkheim (Émile), Les règles de la méthode sociologique, Paris : F. Alban, 1895. Elle a récemment été récusée par l'école de l'individualisme méthodologique.

27. Ceci est bien illustré dans l'analyse d'E. Evans-Pritchard des 13 différents niveaux de conscience spatiale existant parmi la tribu Nuer vivant dans le Haut-Nil. Voir Evans-Pritchard (E.), The Nuer, Oxford : Oxford University Press, 1940, chapitre 3.

28. Weber (Eugen), Peasants in Frenchmen: The Modernisation of Rural France, 1870-1914, Stanford : Stanford University Press, 1976 [La fin des terroirs: la modernisation de la France rurale, 1870-1914, Paris : Gallimard, 1983]. Pour la plupart des habitants, le monde avec lequel ils avaient des liens s'étendait rarement au-delà du village, d'où l'expression l'esprit de clocher, qui correspond au tutejsi polonais.

29. Harvard Encyclopedia of American Ethnic Groups, Cambridge, Mass. : Harvard University Press, 1980.

30. La contradiction entre l'uniformité, telle que suggérée par le modèle totalitaire, et la diversité, dérivée du nationalisme méthodologique, peut expliquer la pénurie et la superficialité des études comparatives sur des États communistes.

31. Un bon exemple de ceci est : Tomaszewski (Jerzy), " The national question in Poland in the twentieth century", in Teich (Mikulas), Porter (Roy), eds., The National Question in Europe in Historical Context, Cambridge: Cambridge University Press, 1993. En dépit du titre, l'analyse s'arrête en 1945, c'est-à-dire lorsque la Pologne devient complètement "polonaise".

32. Voir Zuzowski (Robert), "The impact of nationalism on communism : the case of Poland", Canadian Review of Studies on Nationalism, 1993.

\section{RÉSUMÉS}

Deux paradigmes ont dominé le champ social dans l'analyse occidentale des régimes communistes : le totalitarisme et le libéralisme. Ils ont formé la clef de voûte des explications du nationalisme post-communiste, le "gel communiste » et le "vide idéologique ». Après avoir critiqué ces quatre interprétations des sociétés communistes et post-communistes, l'auteur passe en revue les présupposés sous-tendus par l'ethnocentrisme implicite de ces analyses, d'abord par leur insistance sur la nation comme constituant une réalité donnée, ensuite par leur absence d'intérêt pour l'influence des régimes communistes sur les traditions nationales.

Two paradigms have dominated the social field in Western analysis of communist regimes: totalitarianism and liberalism. They were the keystone of the explanations of the postcommunist nationalism : the «ice-box " approach and the «vacuum » theory. After having criticized these four interpretations of communist and postcommunist societies, the author is being examining the presuppositions underlied by the methodological nationalism of these analysis, first by taking the nation as a given reality, second by lacking of interest in the influence that the communist experience exerted on national traditions. 
INDEX

Mots-clés : Communisme, Nationalisme, Totalitarisme

\section{AUTEURS}

\section{OLIVER FREEMAN}

Oliver FREEMAN est rattaché au St Antony's College à Oxford où il termine sa thèse sur l'évolution du nationalisme dans la Pologne communiste. 